\title{
A Novel Chronic Dural Port Platform for Continuous Collection of Large Volumes of Cerebrospinal Fluid and Intrathecal Drug Delivery in Free-Moving Mice.
}

\section{Tsuneo Nakajima}

Osaka University School of Medicine Graduate School of Medicine: Osaka Daigaku Daigakuin Igakukei Kenkyuka Igakubu https://orcid.org/0000-0002-3670-1701

Shuko Takeda ( $\nabla$ takeda@cgt.med.osaka-u.ac.jp )

Osaka University https://orcid.org/0000-0002-5258-1981

\section{Yuki lto}

Osaka University School of Medicine Graduate School of Medicine: Osaka Daigaku Daigakuin Igakukei Kenkyuka Igakubu

\section{Akane Oyama}

Osaka University School of Medicine Graduate School of Medicine: Osaka Daigaku Daigakuin Igakukei Kenkyuka Igakubu

\section{Yoichi Takami}

Osaka University School of Medicine Graduate School of Medicine: Osaka Daigaku Daigakuin Igakukei Kenkyuka Igakubu

\section{Yasushi Takeya}

Osaka University School of Medicine Graduate School of Medicine: Osaka Daigaku Daigakuin Igakukei Kenkyuka Igakubu

\section{Koichi Yamamoto}

Osaka University School of Medicine Graduate School of Medicine: Osaka Daigaku Daigakuin Igakukei Kenkyuka Igakubu

\section{Ken Sugimoto}

Osaka University School of Medicine Graduate School of Medicine: Osaka Daigaku Daigakuin Igakukei Kenkyuka Igakubu

\section{Hideo Shimizu}

Osaka University School of Medicine Graduate School of Medicine: Osaka Daigaku Daigakuin Igakukei Kenkyuka Igakubu

\section{Munehisa Shimamura}

Osaka University School of Medicine Graduate School of Medicine: Osaka Daigaku Daigakuin Igakukei Kenkyuka Igakubu Hiromi Rakugi 
Osaka University School of Medicine Graduate School of Medicine: Osaka Daigaku Daigakuin Igakukei Kenkyuka Igakubu

\section{Ryuichi Morishita}

Osaka University School of Medicine Graduate School of Medicine: Osaka Daigaku Daigakuin Igakukei Kenkyuka Igakubu

\section{Methodology}

Keywords: cerebrospinal fluid, biomarker, mouse model, CNS disorder, intrathecal space, real-time monitoring

Posted Date: May 19th, 2021

DOl: https://doi.org/10.21203/rs.3.rs-508382/v1

License: (c) (1) This work is licensed under a Creative Commons Attribution 4.0 International License. Read Full License 


\section{Abstract}

\section{Background}

Cerebrospinal fluid (CSF) provides a direct representation of the pathophysiological changes occurring in the central nervous system (CNS); therefore, it has been used for pathogenesis research and biomarker development for CNS disorders. The CSF obtained from valid mouse models relevant to CNS disorders can be an important resource for successful biomarker and drug development. However, the limited volume of CSF that can be collected from the tiny intrathecal space and the technical difficulties involved in CSF sampling have been bottlenecks that have hindered the detailed analysis of CSF in mouse models.

Results

We have developed a novel chronic dural port (CDP) method without cannulation for mouse CSF collection. This method allows easy and repeated access to the intrathecal space in a free-moving, unanesthetized mouse, thereby enabling continuous long-term CSF collection with minimal tissue damage and providing a large volume of high-quality CSF from a single mouse. When combined with chemical biosensors, the CDP method allows real-time monitoring of the dynamic changes in neurochemicals in the CSF at a one-second temporal resolution in free-moving mice. The CDP can also serve as a direct access point for intrathecal injection of CSF tracers and drugs. The spatio-temporal distribution of CSF tracers delivered via CDP revealed a dynamic metabolism of CSF at distinct brain areas. Direct intrathecal delivery of centrally acting drugs via CDP enabled real-time behavioral assessment in free-moving mice.

\section{Conclusions}

The CDP method allows collection of a large volume of high-quality CSF and direct intrathecal drug administration with real-time behavioral assessment in free-moving mice. Combined with animal models relevant to CNS disorders, this method provides a unique and valuable platform for biomarker and therapeutic drug research.

\section{Background}

Cerebrospinal fluid (CSF) provides a direct representation of the pathophysiological changes occurring in the central nervous system; therefore, it has been used for pathogenesis research and biomarker development for central nervous system (CNS) disorders. $(1,2)$ CSF biomarkers have proven useful for the diagnosis and prognosis of many neurodegenerative disorders.(3-5) However, the molecular mechanisms of most CNS disorders remain largely unknown; therefore, the use of mouse models combined with analysis of CSF markers is required to facilitate research.

The mouse is the most commonly used model organism for human disorders and has been served as a useful and adaptable tool for researchers performing basic and preclinical research.(6) A number of 
mouse models relevant to CNS disorders are available and have played a significant role in exploring the molecular mechanisms underlying the disorders, in testing the efficacy of candidate drugs, and in developing CNS biomarkers. $(7,8)$ The CSF obtained from valid mouse models relevant to CNS disorders can be an important resource for successful biomarker research; however, the ability to collect only a very limited volume of CSF from a mouse and the technical difficulties related to CSF collection procedures have been bottlenecks that have hindered the detailed analysis and comprehensive profiling of metabolites and proteins in the CSF. (9)

Three basic methods have been reported and used for mouse CSF collection. $(9,10)$ The most commonly used technique is a single collection by cisterna magna (CM) puncture, in which CSF is withdrawn using a pipette or fine glass capillary via a small hole made in the membrane covering the CM.(9-11) This method has been widely used for mouse CSF research; however, the amount of CSF obtained is relatively small ( $10 \mu$ l per single mouse), and the procedure requires anesthesia and head fixation, which prevents CSF sampling under physiological conditions and could affect the quality and quantity of the collected CSF. Repeated serial collection from the same mouse is also difficult with this method. Another method is intrathecal cannulation, which involves insertion of a fine cannula into the intrathecal space.(12) This method allows repeated collection at different time points from the same mouse; however, tissue injury due to cannula insertion into the narrow intrathecal space of mouse is the main drawback of this method. $(13,14)$ The use of this method can be especially problematic when the collected CSF is intended for use in biomarker research targeting neurodegenerative marker proteins, such as tau and neurofilament.(10) A third option is CSF collection from the lateral ventricle via a needle stereotaxically targeted to the ventricle.(15) This method can be used for CSF collection as well as for drug administration directly into brain; however, it requires penetration of the needle through the brain parenchyma, thereby increasing the susceptibility to an artifactual increase in neurodegenerative markers.(10)

An ideal method for CSF collection from mice has some specific requirements, as it must allow: (1) collection of a large volume of CSF from a single mouse, (2) a long-term continuous collection from the same animal, (3) CSF collection under physiological conditions in an awake, free-moving mouse, and (4) repeated serial collections at different time points over time. This ideal CSF collection method would enable a detailed comprehensive profiling of components of the CSF, the monitoring of longitudinal changes in biomarker levels, and determination of the effects of centrally acting drugs. The choice of method is also important from a viewpoint of animal ethics, so an ideal method should also reduce the number of mice needed for CSF experiments.

Methods adopted for CSF collection can also be used for drug or tracer administration into the intrathecal space.(12) The penetration of most drugs is restricted by the blood-brain barrier (BBB), so many small drug compounds and proteins cannot enter the CNS.(16) Intrathecal administration therefore has advantages over other drug delivery procedures, such as oral and intravenous administrations, as it reduces side effects in the peripheral tissues and provides direct and rapid exposure of the drug to the CNS. Intrathecal delivery has been used for some CNS disorders in clinical settings, (12) and its use has been reported for drug administration in mice; however, this method poses a risk of tissue injury due to 
cannula insertion.(12) Therefore, development of method for stable and repeated intrathecal administration with minimal risk of tissue damage would be useful for therapeutic development and for evaluating the dynamic metabolism of CNS tracers.

In the present study, we describe the development of a chronic dural port (CDP) method as a novel approach for mouse CSF collection and intrathecal drug delivery. The CDP is installed on the CM, together with CSF collection tubing fixed using dental cement. This system allows easy and repeated access to the CSF, and connection to a roller/syringe pump enables continuous long-term CSF collection in a freemoving, unanesthetized mouse. The method allows collection of a large volume of high-quality CSF from a single animal. The CDP can also serve as a direct access point to the intrathecal space, thereby allowing direct intrathecal drug administration and real-time behavioral assessment in free-moving mice.

\section{Results}

\section{Chronic dural port for continuous collection of CSF in a free-moving mouse.}

Fig. 1 shows the schematic for continuous CSF collection using the CDP method in an awake, freemoving mouse. The CDP is installed on the cisterna magna (CM) at the dorsal part of mouse's neck. The CSF collection tubing from the CDP is connected to a roller pump (or syringe pump) to withdraw CSF at a constant flow rate (Fig. 1a, left). This method does not require anesthesia for the mouse during an experiment, thereby enabling continuous long-term collection of CSF under ad libitum conditions for water and food intake. The mouse is tethered with the sensor-integrated balance arm and placed into the movement-response caging system (17) designed to allow unrestricted movement of the animal without applying pressure to the probe assembly (Fig. 1a, right).

The detailed structure of the CDP is shown in Fig. 1b. A small hole is made on the atlanto-occipital membrane (AOM) covering the $\mathrm{CM}$ and connected to the CSF collection tubing. The size of hole on the AOM $(\sim 0.2 \mathrm{~mm})$ is made slightly smaller than the internal diameter of the CSF collection tubing $(0.3 \mathrm{~mm})$ to prevent leakage of the CSF during collection. The CSF is collected by applying negative pressure to the CSF collection tubing with a roller (or syringe) pump. Dental cement is used to fix the CSF collection tubing, the AOM, and the surrounding bone structures, including the occipital crest and the anterosuperior border of the atlas, thereby allowing stable collection of CSF without leakage in the free-moving mouse. Notably, the tip of the CSF collection tubing is not inserted into the intrathecal space; instead, it is attached and fixed onto the AOM covering the small hole (Fig. 1b). This is an important feature of the CDP method as it prevents tissue damage during the long-term continuous CSF collection in the freemoving mouse (Fig. 2).

The CDP method enables the collection of a large volume of CSF from a single mouse. The conventional methods, like single collection via a CM puncture, allows collection of only a relatively small volume ( 10 $\mu \mathrm{l})$ of CSF (Fig. 1c, left). $(11,18)$ Continuous CSF collection via the CDP allows collection of approximately $500 \mu \mathrm{l}$ of CSF from a single mouse over a five-day collection period when the flow rate is maintained at $0.067 \mu \mathrm{l} / \mathrm{min}$ (Fig. 1c, right). This sampling flow rate is much lower than the physiological 
production rate $(\sim 0.35 \mu \mathrm{l} / \mathrm{min})$ of mouse $\operatorname{CSF}(1,19,20)$. Since the continuous CSF collection via the CDP can be conducted in a free-moving mouse given ad libitum access to water, the mouse can maintain its physiological condition as the rate of CSF sampling is sufficiently lower than the physiological production rate.

\section{Continuous collection of high-quality CSF from naturally behaving mice.}

We also investigated the quality of the CSF collected via the CDP. Blood contamination in the CSF can have a significant impact on the analytic outcome in biomarker research (21). A high-sensitivity spectrophotometry method (10) was used to measure blood contamination in the collected CSF. This method allows detection of blood contamination down to $0.001 \%$, a level undetectable by eye. As shown in the inset photos in Fig. 2 a, contamination with $0.1-1 \%$ blood was visible, while blood contamination lower than $0.01 \%$ was undetectable to the naked eye. Continuously collected CSF (cCSF) via CDP had a clear appearance, and blood contamination was undetectable by the high-sensitivity spectrophotometer method, indicating that the collected CSF was as clean as CSF collected via a conventional singlecollection technique (Fig. 2a). Notably, no significant blood contamination was detected in the cCSF during long-term collection over a few days. We also measured the levels of basic CSF electrolytes, such as sodium, potassium, and chloride, in the CSF collected by a conventional single-collection method and the CDP method (CCSF). The concentrations of these electrolytes in the cCSF were maintained within the physiological range (22) over the three-day collection and were comparable to those determined in CSF obtained by a single collection (Fig. 2b).

We also investigated the impact of continuous CSF collection via the CDP on mouse behavior. The locomotor activity of the animal was quantitatively measured using the infrared beam-based activity detection system.(23) Baseline measurements were obtained for two days, and then the continuous CSF collection was started in the middle of light phase of the second day (12:00 on day 2) and continued for two more days (Fig. 2c). The mice under continuous CSF collection maintained a normal circadian rhythm, with no significant differences in the dark/light ratio of locomotor activity between baseline and during the cCSF collection (Fig. 2d. This suggested that continuous CSF collection via CDP has no impact on the physiological behavior of the mice.

\section{Minimal tissue damage on brain and spinal cord after long-term CSF collection via CDP}

CSF collection via conventional CMc can cause tissue damage after a long-term experiment lasting over several days or weeks, especially when using small-sized rodent like a mouse. Tissue damage due to the cannula tip inserted into the intrathecal space can cause behavioral impairments and increases in biomarkers of neurodegeneration, such as neurofilament and tau protein (10), which may distort the conclusions of the experiment. Indeed, placement of an intrathecal catheter in the intrathecal space of rat has been reported to cause significant damage and inflammation to the central nervous system. $(13,14)$ The probability of tissue damage is higher when using a mouse model due to the smaller intrathecal space. 
In contrast to the conventional CMc method, the CDP does not require intrathecal insertion of a cannula for CSF collection (Fig. 3a) and is thereby expected to reduce tissue damage. Fig. 3b-d shows a sagittal section of the brain and spinal cord seven days after the surgery for CDP installation). No tissue injury or inflammation is apparent with the CDP method, whereas the CMc method shows clearly visible injury on the surface of the brain stem (Fig. 3b); this injury was also accompanied by excessive accumulation of GFAP-positive astrocytes (Fig. 3c). The number of GFAP-positive astrocytes in the brain tissue underneath the $\mathrm{CM}$ was significantly higher with the $\mathrm{CMc}$ method, whereas the mice subjected to the CDP method had comparable GFAP-positive astrocytes numbers to those in naïve mice (Fig. $3 d$ ). These results mean that the CDP method enables a long-term CSF collection from mice with minimal tissue damage.

\section{Application of the CDP method for continuous real-time glucose monitoring in the CSF}

When combined with biosensors for specific molecules, the CDP method allows continuous monitoring of the dynamic changes of the molecules in the CSF in free-moving mice. We applied the CDP method for monitoring the dynamic metabolism of glucose and lactate in the CSF during peripheral glucose loading. To do this, we used enzyme-based biosensors, which enable second-by-second recording of the concentration changes of each molecule (Fig. 4). The biosensors for glucose and lactate were attached in the middle of the CSF collection tubing (biosensor port); each biosensor electrode was connected to the recording software with an amplifier (Fig. 4a). (24) The mouse was housed in the movement-response caging system, which allows free movement during the experiment. Fig. $4 \mathrm{~b}$ shows the structure of the biosensor port in which electrodes are exposed to the continuous flow of CSF to monitor real-time changes in glucose and lactate concentrations. The inlet of the port is connected to the CDP and the outlet is connected to a syringe pump to withdraw CSF at a constant flow rate.

The mouse was given an intraperitoneal injection of glucose during continuous CSF collection at a flow rate of $0.05 \mu \mathrm{l} / \mathrm{min}$. The CSF glucose concentration showed a rapid increase after intraperitoneal glucose loading, reaching its peak at $\sim 50 \mathrm{~min}$, followed by a gradual decrease down to baseline level at $\sim 180 \mathrm{~min}$ (Fig. 4c). The CSF lactate concentration did not show any significant change during glucose loading (Fig. $4 \mathrm{c}$ ), indicating the specificity of each biosensor for each molecule. The CDP method for continuous CSF collection, combined with the glucose/lactate biosensors, allows real-time monitoring of dynamic glucometabolic changes in the central nervous system at a one-second temporal resolution in freemoving mice.

\section{Injection of a CSF tracer via the CDP and its temporal and spatial distributions in mice.}

The CDP can also be used as an injection port for various molecules, such as CSF tracers and drugs. We investigated the spatio-temporal distribution of CSF tracers injected into the intrathecal space via the CDP (Fig. 5). A contrast agent used for micro-computed tomography (micro-CT) imaging was injected via the CDP, followed by sequential CT imaging of the mouse brain for 90 min (Fig. 5a-e). The CT images of five coronal sections from different parts of the mouse brain, at the levels of cribriform plate, hypothalamus, cerebral aqueduct, fourth ventricle, cisterna magna, and spinal cord (Fig. 5b), were used for quantitative measurement of the concentrations of the injected contrast agent (Fig. 5c). The contrast agent injected 
via the CDP showed quick and broad rostral-to-caudal distribution from the levels of the cribriform plate to the spinal cord sections (Fig. 5c).

We also evaluated the dynamic metabolism of the injected tracer at each part of the intrathecal space by measuring the temporal changes in the concentrations and calculating the half-life (t1/2) and the time to peak concentration (Tmax) values (Fig. $5 d, e$ ). In the areas adjacent to the infusion site (CDP), such as the $\mathrm{CM}$ and the 4th ventricle, the injected tracer immediately reached its peak concentration (Tmax, 10 min), followed by a rapid decrease to the baseline levels by $60 \mathrm{~min}(\mathrm{t} 1 / 2,<3 \mathrm{~min}$ ) (Group $\mathrm{A}$ in Fig. 5e). At the levels of the hypothalamus and the cerebral aqueduct, the injected tracer reached its peak a few minutes later (Tmax, 10-20 min), followed by a gradual decrease to the baseline levels by $60 \mathrm{~min}(\mathrm{t} 1 / 2, \sim 5 \mathrm{~min})$ (Group B in Fig. 5e). In the areas distant from the infusion site, like the cribriform plate and the spinal cord, the injected tracer took longer to reach its peak levels (Tmax, $>30 \mathrm{~min}$ ) and to wash out $(\mathrm{t} 1 / 2,7-8$ min) (Group $\mathrm{C}$ in Fig. 5e). The difference in the kinetic parameters (t1/2 and Tmax) among the distinct intrathecal spaces suggests that the dynamic metabolism and circulation of the CSF differs at each area. This should be taken into consideration when interpreting data regarding CSF biomarkers and the pharmacokinetics of drugs in the CSF.We also evaluated the spatial distribution and the degree of penetration of a tracer injected into the brain parenchyma via the CDP by histological assessment (Fig. $5 f, g$ ). We injected a single dose of a water-soluble nuclear-staining dye (Hoechst) via the CDP, harvested the brain at $60 \mathrm{~min}$, and examined the nuclear staining in brain sections taken from different areas (Fig. 5f). A broad distribution of the injected dye was noted, ranging from the most rostral part of the olfactory bulb (at the levels of Bregma $3.0 \mathrm{~mm}$ ) to the most caudal part of the spinal cord (at the levels of the cervical C3), with the highest staining intensity at the injection site of the CM (Fig. 5f, top panels). Notably, the dye injected retrogradely into the CM passed to the 4th ventricle (Fig. $5 f$, indicated as dotted rectangle 3 ), through the cerebral aqueduct (Fig. $5 f$, indicated as dotted rectangle 2), and then to the 3rd ventricle (Fig. 5f, indicated as dotted rectangle 1). Nuclear staining was observed in the cells of the hippocampal dentate gyrus and the subiculum (Fig. 5f, lower panel (1)), indicating that these brain areas can be exposed to a drug injected via the CDP into the CM. Fig. $5 \mathrm{~g}$ shows a coronal section at the level of the dorsal hippocampus (Bregma $-2.5 \mathrm{~mm}$ ) after immunostaining with the neuronal cell marker NeuN. The nuclear staining was stronger in the ventral part of the brain than in any of the other regions when the dye was injected into $\mathrm{CM}$ with the mouse placed in the normal prone position (Fig. $5 \mathrm{~g}$, indicated as dotted rectangle 1), suggesting that an intrathecally injected tracer tends to be retained in the basal part of the brain. Deep penetration of the tracer into the brain parenchyma was observed at the ventral part of the brain, extending to $\sim 400 \mu \mathrm{m}$ from the surface (Fig. $5 \mathrm{~g}$, indicated as dotted rectangle 1 ). The lateral ( $\sim 300 \mu \mathrm{m}$ depth) and dorsal ( 100 $\mu \mathrm{m}$ depth) parts of the brain showed a more superficial penetration of the tracer (Fig. 5g, indicated as dotted rectangles 2 and 3 , respectively). These findings are important when considering the effect of intrathecally administered drugs, since the difference in the degree of parenchymal penetration among distinct brain areas can have a significant impact on the effect of the drugs.

\section{Application of the CDP method for intrathecal administration of a drug and real-time behavioral assessment.}


The CDP method enables intrathecal administration of centrally acting drugs and real-time assessment of behavioral changes in awake, free-moving mice. We injected a single acute dose of anesthetic agent via the CDP while quantitatively measuring the animal's locomotor activity with high-temporal resolution by an infrared light beam crossing system (Fig. 6a). The mice showed rapid decreases in locomotor activity immediately after administration of the anesthetic agent (within $2 \mathrm{~min}$ ), suggesting a direct pharmacological action of the intrathecally administered drug. The locomotor activity of the animals started to increase again at $6 \mathrm{~min}$ after drug administration, indicating a relatively fast recovery from the anesthetic to the awake state (Fig. 6b). Intrathecal drugs can be administered by the CDP without head fixation of behaving mice, thereby providing advantages in behavioral assessment when testing the effects of drugs on cognition and behavior.

\section{Discussion}

Brain tissue is surrounded and protected by a bony structure, which makes it difficult to perform biopsy or direct delivery of therapeutic drugs; therefore, CSF collection or intrathecal drug administration has been used as an alternative method. The small size of the intrathecal space in mice disables stable collection of a large volume of CSF for detailed analysis of its components, which has been the main bottleneck for CSF biomarker research using mouse models. Herein, we developed a CDP method as a novel approach for CSF collection that allows continuous long-term CSF sampling in awake, free-moving mice. This method enables collection of large volumes of high-quality CSF suitable for biomarker research and can also be used for intrathecal delivery of drugs for real-time monitoring of their pharmacological effect on the behavior of free-moving mice.

The volume of CSF obtained through conventional methods such as CM puncture ( $10 \mu \mathrm{l}$ per single mouse) $(9,10)$ is not sufficient for the measurement of multiple biomarkers even when using a highly sensitive ELISA assay and requires pooling of CSF from multiple animals to obtain a large volume for measurement. The CDP method allows continuous long-term collection of CSF from a single mouse; therefore, a large volume of CSF sufficient for comprehensive profiling of CSF components can be obtained. Sampling can be performed under ad libitum conditions for water and food intake, which prevents dehydration during continuous long-term CSF collection. The flow rate of the CSF collection used in this study $(<0.07 \mu \mathrm{l} / \mathrm{min})$ was lower than the physiological production rate of mouse CSF $(\sim 0.35$ $\mu \mathrm{l} / \mathrm{min})(1,19,20)$. We confirmed that long-term CSF collection at this flow rate does not have a significant impact on the electrolyte concentrations of CSF (Fig. 2b) and the physiological behavior of mice, such as diurnal circadian rhythm (Fig. 2c, d). Since CDP enables obtaining large volumes and repeated serial collection from a single mouse, it can be useful for monitoring long-term changes in biomarker levels and the efficacy of therapeutic intervention over time, which can reduce the number of animals needed for the experiment.

The quality of collected CSF is critical, especially for biomarker development research $(10,21)$. Blood contamination in CSF significantly impacts biomarker analysis, especially when the concentration of the molecule is supposed to be higher in blood than in CSF like alpha-synuclein (21). In addition, blood 
contamination can increase non-specific noise reaction due to a large number of hydrophobic immunoglobulin components, resulting in sample-related biases (25). Tissue injury during CSF collection or intrathecal drug delivery, especially when using the intrathecal cannulation method, could increase the concentrations of neurodegenerative markers such as tau and neurofilament as an artifact. Notably, the CDP method does not need the CSF collection tubing inserted into the intrathecal space and therefore significantly reduces the probability of tissue injury even in long-term experiments. The large volume of CSF collected via CDP was free of detectable blood contamination (< $0.01 \%)$ (Fig. 2a) and caused minimal tissue damage and accumulation of inflammatory cells (Fig. 3 ).

The main mechanisms of CSF production, circulation, and absorption, as well as the dynamic metabolism of CSF components including proteins, electrolytes, and neurochemicals, are still largely unknown (26). The knowledge of these fundamental aspects of the CSF metabolism is essential for biomarker development and for understanding the pathogenesis of CNS disorders related to CSF dysregulation such as normal pressure hydrocephalus (NPH) (27). Continuous collection of CSF via CDP combined with specific and sensitive biosensors enables real-time measurement of dynamic changes in the CSF components of free-moving mice (Fig. 3), which provides a useful platform for basic research on the CSF metabolism. To the best of our knowledge, this is the first report to demonstrate the monitoring of dynamic changes in CSF glucose levels at one-second temporal resolution in free-moving mice (Fig. 3c). Sensitive measurement of CSF glucose and other neurochemicals at a high temporal resolution can be a valuable tool for understanding pathophysiological changes in the glucometabolic state in CNS disorders $(28,29)$.

The CDP method can also be applied to intrathecal drug delivery for therapeutic development. Intrathecal administration is used in the treatment of several CNS disorders such as pain, multiple sclerosis, and brain tumor (30-32) because this approach can bypass the BBB and enable direct pharmacological action on the CNS. Here, we demonstrated that the CDP method combined with a behavioral assessment tool, such as an activity cage for recording spontaneous coordinate activity, enables real-time evaluation of a centrally acting drug delivered into the intrathecal space in free-moving mice (Fig. 6). Intrathecal drug delivery via CDP in free-moving mice with minimal physical restraint has key advantages over conventional methods such as intrathecal canulation $(13,14)$ or direct injection into the latera ventricle (15) in which tissue injury-associated motor or cognitive impairment can be a serious problem in behavioral assessment.

Using the CDP method, we revealed the spatio-temporal distribution and metabolism of an intrathecally delivered CNS tracer (Fig. 5). The CNS tracer injected into CM via CDP was widely distributed in the intrathecal space and, notably, reached to hippocampal area (Fig. 5f). However, there was a significant difference in the kinetic parameters, such as t1/2 and Tmax, of the brain regions (Fig. 5d, e), suggesting the complexity of CSF circulation and metabolism. This should be taken into consideration when evaluating the therapeutic effect of intrathecally administered drugs. 
The drawbacks of the CDP method include (1) potential limitation of neck movement due to CDP fixation with dental cement and (2) requirement for surgical training. The CDP method requires fixation of the CSF collection tubing with the AOM and surrounding bone structures including the occipital crest and anterosuperior border of the atlas, which is important for stable CSF collection in free-moving mice. We did not observe apparent behavioral alterations after CDP installation. Mice with CDP showed rapid recovery from the surgery, and surgery-related body weight loss was restored within four days after operation (data not shown). Notably, the mice with CDP implantation survived as long as a few months after surgery, indicating the minimal physical stress this method exerts on animals; the mice in this study maintained normal circadian rhythm even during continuous CSF collection (Fig. 2c, d). Although this surgical procedure requires training, a basic animal surgery setup is sufficient, and the step-by-step instructions described in the method section should be helpful for researchers to master the technique. In our experiment, the mortality of animals after CDP surgery was less than $2 \%$ (data not shown).

\section{Conclusions}

The CDP method provides a unique and valuable platform for CSF biomarker research and therapeutic development using animal models relevant to CNS disorders. An implanted CDP allows repeated and long-term access to CSF and the intrathecal space in awake, free-moving mice. A large volume of highquality CSF collected from mice under physiological conditions can be useful to identify novel relevant biomarkers.

\section{Methods}

\section{Animals}

All animal experiments were performed in compliance with the Guidelines for the Care and Use of Laboratory Animals at Osaka University School of Medicine and the Guidelines of the NIH for the Care and Use of Laboratory Animals. Three- to four-month-old male mice (C57BL6/J) weighing 20-25 g were used in the study (obtained from CLEA Japan). Animals were maintained at room temperature $\left(25 \pm 2{ }^{\circ} \mathrm{C}\right)$ under a standard 12-h/12-h light-dark cycle, with free access to water and food.

\section{Surgical procedure for CDP implantation}

The CSF collection tubing for CDP (M025V-100, EICOM, Japan), made of polyurethane (0.3 mm i.d.), was cut to a length of $3 \mathrm{~cm}$ via a razor blade. The CSF collection tubing was connected to the FEP tube with joint (JF-10, \#800160, EICOM, Japan), which was connected to a peristaltic pump (ERP-10, \#600100, Eicom, Japan) or microsyringe pump (ESP-64, Eicom, Japan). The tubing was filled with distilled water prior to surgery.

Mice were anesthetized with an intraperitoneal injection of a combination of domitor $(0.75 \mathrm{mg} / \mathrm{kg})$, midazolam (4 mg/kg), and butorphanol $(5 \mathrm{mg} / \mathrm{kg})$, and then mounted on a stereotaxic frame (SR-5M-HT, NARISHIGE, Japan), with a heating pad placed underneath the body. The mouse is laid down so that the 
head approximately forms an 80-degree angle with the body, secured by using the head adaptor. The surgical area was disinfected with iodine and covered by a surgical drape. Using straight-tip surgical scissors, an incision $(20 \mathrm{~mm})$ in the skin was made at the center of the neck. Under the dissection microscope, the subcutaneous tissue and muscle layers were separated by blunt dissection with forceps and fine-tipped cotton swabs to expose the AOM of CM.

A small hole was made in the center of the AOM by shallow centesis using a $30 \mathrm{G}$ needle, with caution taken to avoid damage to brain tissue and blood vessels. After confirming the CSF flowing out of the puncture due to cerebrospinal pressure, the tip of the CSF collection tubing was attached to the membrane by folding the tubing with a stereotactic arm so that it covered the small hole made on the AOM. Then the CSF was withdrawn via a peristaltic pump at a flow rate of $20 \mu \mathrm{l} / \mathrm{hour}$, which visually confirmed that the CSF was being drained into the cannula. After confirming this, the flow rate was decreased to $10 \mu \mathrm{l} / \mathrm{hour}$.

The CSF collection tubing, AOM, and surrounding bone structures, including the occipital crest and anterosuperior border of the atlas, were fixed with 4-META/MMA-TBB resin (Super-bond C\&B, Sun Medical, Japan). After confirming that the CSF continued to be drained and the cement was completely secured, the flow rate was further decreased to $4 \mu \mathrm{l} / \mathrm{hour}$.

The CSF collection tubing was disconnected from the FEP tube, and the outlet was plugged. The skin was aligned and sutured using 7-0 silk, then a mixture of analgesic, sugar, and antibacterial agents were administered intraperitoneally. The mouse was left on top of the heating pad to maintain its body temperature until it awoke. The mice were then carefully transferred to a recovery home cage. Furthermore, they were allowed at least four days for tissue recovery before starting continuous CSF collection.

\section{Continuous CSF collection via CDP}

Four days after the implantation of CDP, the mice were tethered with the sensor-integrated balance arm and placed into the movement-response caging system (MD-1409, BASi, USA as described previously (17). The sensor detects the rotation of the animal and turns the cage in the opposite direction, which allows unrestricted movement of the animals without applying pressure to the probe assembly. The CSF collection tubing (M025V-100, Eicom, Japan) from the CDP is connected to a roller pump (ERP-10, Eicom, Japan) or syringe pump (ESP-32, Eicom, Japan) to withdraw CSF at a constant flow rate. The movementresponse caging system was placed in the sound-attenuating box (Natsume Seisakusho, Osaka, Japan) to control environmental conditions; the mice were maintained at room temperature $\left(25 \pm 2{ }^{\circ} \mathrm{C}\right)$ under a standard 12-h/12-h light-dark cycle, with free access to water and food. The locomotor activity of the mice during the experiment was measured by an infrared light beam crossing system as described below.

\section{A single-collection technique for mouse CSF via CM puncture}


A conventional single-collection technique for mouse CSF was performed as previously described, with minor modifications (10). Mice were anesthetized with an intraperitoneal injection of a combination of domitor $(0.75 \mathrm{mg} / \mathrm{kg})$, midazolam (4 mg/kg), and butorphanol $(5 \mathrm{mg} / \mathrm{kg})$, then placed on a warmed surgical platform. Under the dissection microscope, the subcutaneous tissue and muscles are separated to expose the CM. CSF was collected by puncturing the AOM with a $30 \mathrm{G}$ needle and aspirated with a P20 pipettor.

\section{Chronic CMc method}

For the chronic CMc experiment, implantation of a catheter into the cisterna magna in mice was performed as described earlier (12). Three days after CMc surgery, the mice were euthanized, and their brains were harvested for histology to evaluate the tissue damage due to the intrathecally inserted cannula.

\section{Assessment of blood contamination and measurement of electrolytes in CSF}

A high-sensitivity spectrophotometry method was used to measure blood contamination in the collected CSF, as described previously (10). Levels of hemoglobin in the collected CSF, released by the hypotonic freeze-thaw method, were quantified by measuring the absorbance at $417 \mathrm{~nm}$ on a NanoDrop ND-1000 spectrophotometer (NanoDrop Technologies/Thermo Scientific, Wilmington, DE, USA). We used clear CSF spiked with increasing amounts of whole blood $(0.01 \%, 0.1 \%$, and $1 \%)$ as references. Pooled CSF was centrifuged at 2,000 $\mathrm{g}$ for 30 seconds to remove any red blood cells, and the supernatant was used for clear CSF. The concentrations of electrolytes (sodium, potassium, and chloride) in the collected CSF were measured by a blood gas analyzer (Radiometer ABL 700, Radiometer Medical, , Denmark)

\section{Biosensor measurements of glucose and lactate in CSF}

Enzyme-based biosensors were used for real-time monitoring of glucose and lactate levels as previously described $(24,33)$. In this study, the distal end of the electrodes of glucose and lactate biosensors (Part \#7004-80-Glucose and \#7004-80-Lactate; Pinnacle Technology, U.S.A.) were inserted in the biosensor port (Eicom, Japan), where the biosensors were exposed to the continuously collected CSF. All biosensors were calibrated in vitro prior to implantation. Mice were given a single dose of an intraperitoneal injection of glucose ( $2 \mathrm{~g} / \mathrm{kg}$ body weight) during biosensor measurements of CSF glucose and lactate.

\section{Brain histology}

Brains were fixed in $4 \%$ PFA for 24 hours at $4{ }^{\circ} \mathrm{C}$, incubated for 2 days in $30 \%$ sucrose in PBS and embedded in a Tissue-Tek O.C.T. Compound (Sakura Finetek Japan Co., Ltd.), and then the sagittal and coronal sections of $8 \mu \mathrm{m}$ thickness were cut on a cryostat and mounted on glass slides. Brain sections were stained with hematoxylin and eosin (H\&E) to evaluate the tissue integrity and damage due to CSF collection. For immunostaining, sections were permeabilized with $0.5 \%$ Triton X-100 in PBS, blocked in 5\% BSA in PBS, and immunostained with rabbit anti-GFAP antibody (1:200, PRB-571C, BioLegend) and mouse anti-NeuN antibody (1:200, MAB377, Merck) in 1\% BSA in PBS at RT overnight. Sections were 
washed thoroughly in PBS, and then immunoreactions were visualized by fluorescent secondary antibodies. Sections were mounted using VECTASHIELD medium ( $\mathrm{H}-1000$, Vector Laboratories). We used the following secondary antibodies: Alexa 488-conjugated goat anti-rabbit IgG antibody (1:200, ab150077, abcam) and Alexa 594-conjugated goat anti-mouse IgG antibody (1:200, ab150116, abcam). Fluorescent images were captured with a fluorescence microscope (BZ-9000, Keyence, Japan) equipped with a digital camera, using the same fluorescence settings in all cases. The pixel intensities of the fluorescent signal were analyzed and quantified using National Institutes of Health Image software. For GFAP burden analyses, data was reported as the percentage of the labeled area captured (positive pixels) divided by the full area captured (total pixels).

\section{Drug and tracer injection via CDP}

Intrathecal injection of drug and CSF tracer injection was performed by using a 30G Hamilton microsyringe, which was connected to a CDP via CSF collection tubing. The dead volume of CSF collection tubing was approximately $0.6 \mu$ l. A single dose of contrast agent ( $5 \mu$ in 10 seconds) was injected via CDP, followed by sequential micro-CT imaging (R_mCT2, Rigaku Corp., Japan).

Concentrations of contrast agents at each brain region at each time point were determined based on the signal intensity, which were used for calculating metabolic parameters such as $\mathrm{t} 1 / 2$ and Tmax. For histological assessment, a single dose of a water-soluble nuclear-staining dye (Hoechst 33342, invitrogen, USA) was injected via the CDP (50 $\mu \mathrm{g} / 5 \mu \mathrm{l}$ in 10 seconds). The brain was harvested at 60 min. Coronal sections were immunostained with an anti-NeuN antibody as described above, and fluorescent images were captured with a fluorescence microscope (BZ-9000, Keyence, Japan). For behavioral assessment of an intrathecally delivered drug, an anesthetic agent (midazolam, Maruishi Pharmaceutical, Japan) was acutely injected via CDP in the free-moving mice $(25 \mu \mathrm{g} / 5 \mu \mathrm{l}$ in $1 \mathrm{sec}$ by a syringe connected to the CDP), while locomotor activities were measured by an infrared light beam crossing system as described next. Following a baseline measurement for $5 \mathrm{~min}$, an anesthetic agent was acutely delivered, and locomotor activity recording continued until $10 \mathrm{~min}$.

\section{Behavioral and locomotor measurements using activity monitoring system}

Spontaneous locomotor activity of mice during the continuous CSF collection or intrathecal drug delivery experiment was monitored in an open-filed cage $(43 \mathrm{~cm}$ to $43 \mathrm{~cm})$ with high-density arranged infrared sensors (Scanet, MV-40, Melquest, Toyama, Japan). $(23,34)$ The infrared sensors are distributed in all directions parallel to the floor (37.5 $\mathrm{mm}$ from the floor). The mice were allowed to move freely on the floor. Mice movements were detected by infrared sensors and recorded every $0.1 \mathrm{~s}$.

\section{Statistical analysis.}

All data were expressed as the mean \pm s.e.m. Two-group comparisons were performed by an unpaired ttest, unless stated otherwise. Comparison among three or more groups was performed by analysis of variance and the Tukey-Kramer test, unless stated otherwise. P values $<0.05$ were considered significant. 


\section{Abbreviations}

AOM: atlanto-occipital membrane

BBB: blood-brain barrier

CDP: chronic dural port

CM: cisterna magna

CNS: central nervous system

CSF: cerebrospinal fluid

cCSF: continuously collected CSF

CT: computed tomography

NPH: normal pressure hydrocephalus

Tmax: time to peak concentration.

\section{Declarations}

\section{Ethics approval and consent to participate}

All animal experiments were performed in compliance with the Guidelines for the Care and Use of Laboratory Animals at Osaka University School of Medicine and the Guidelines of the NIH for the Care and Use of Laboratory Animals.

\section{Consent for publication}

Not applicable

\section{Availability of data and materials}

The datasets used and/or analysed during the current study are available from the corresponding author on reasonable request.

\section{Competing interests}

The authors declare no competing interest.

\section{Funding}


This work was supported by JSPS KAKENHI Grant Number 17 H05080 (grant-in-Aid for Young Scientists (A)).

Author information

Affiliations

Department of Geriatric and General Medicine, Graduate School of Medicine, Osaka University, Suita, Osaka, 565-0871, Japan.

Tsuneo Nakajima, Akane Oyama, Yoichi Takami, Yasushi Takeya, Koichi Yamamoto, Ken Sugimoto, Hiromi Rakugi

Department of Clinical Gene Therapy, Graduate School of Medicine, Osaka University, Suita, Osaka, 5650871, Japan.

Shuko Takeda, Yuki Ito, Hideo Shimizu, Ryuichi Morishita

Department of Internal Medicine, Osaka Dental University, Hirakata, Osaka 573-1121, Japan

Hideo Shimizu

Department of Neurology, Department of Health Development and Medicine, Osaka University, Suita, Osaka 565-0871, Japan

Munehisa Shimamura

Osaka Psychiatric Medical Center, Osaka Psychiatric Research Center, Hirakata, Osaka 573-0022, Japan

Shuko Takeda

Department of Clinical Nursing Division of Health Sciences Graduate School of Medicine, Osaka University, Suita, Osaka, 565-0871, Japan

Yasushi Takeya

General and Geriatric Medicine, Kawasaki Medical School General Medical Center, Okayama, 700-8505, Japan

Ken Sugimoto

Contributions

T.N., S.T., H.R., and R.M. designed research; T.N. and S.T. performed research; T.N. and S.T. analyzed data; and T.N. and S.T. wrote the paper. All authors read and approved the final manuscript. 
This work was supported by JSPS KAKENHI Grant Number 17H05080 (grant-in-Aid for Young Scientists (A)).

\section{References}

1. Johanson CE, Duncan JA, 3rd, Klinge PM, Brinker T, Stopa EG, Silverberg GD: Multiplicity of cerebrospinal fluid functions: New challenges in health and disease.Cerebrospinal fluid research 2008, 5:10.

2. Sakka L, Coll G, Chazal J: Anatomy and physiology of cerebrospinal fluid.European annals of otorhinolaryngology, head and neck diseases 2011, 128:309-316.

3. Olsson B, Lautner R, Andreasson U, Öhrfelt A, Portelius E, Bjerke M, Hölttä M, Rosén C, Olsson C, Strobel G, et al: CSF and blood biomarkers for the diagnosis of Alzheimer's disease: a systematic review and meta-analysis. The Lancet Neurology 2016, 15:673-684.

4. Olsson B, Portelius E, Cullen NC, Sandelius Å, Zetterberg H, Andreasson U, Höglund K, Irwin DJ, Grossman M, Weintraub D, et al: Association of Cerebrospinal Fluid Neurofilament Light Protein Levels With Cognition in Patients With Dementia, Motor Neuron Disease, and Movement Disorders.JAMA neurology 2019, 76:318-325.

5. Takeda S, Commins C, DeVos SL, Nobuhara CK, Wegmann S, Roe AD, Costantino I, Fan Z, Nicholls $\mathrm{SB}$, Sherman AE, et al: Seed-competent high-molecular-weight tau species accumulates in the cerebrospinal fluid of Alzheimer's disease mouse model and human patients. Annals of neurology 2016, 80:355-367.

6. Rosenthal N, Brown S: The mouse ascending: perspectives for human-disease models. Nature cell biology 2007, 9:993-999.

7. Onos KD, Uyar A, Keezer KJ, Jackson HM, Preuss C, Acklin CJ, O'Rourke R, Buchanan R, Cossette TL, Sukoff Rizzo SJ, et al: Enhancing face validity of mouse models of Alzheimer's disease with natural genetic variation.PLoS genetics 2019, 15:e1008155.

8. Myers A, McGonigle P: Overview of Transgenic Mouse Models for Alzheimer's Disease.Current protocols in neuroscience 2019, 89:e81.

9. Šakić B: Cerebrospinal fluid collection in laboratory mice: Literature review and modified cisternal puncture method.Journal of neuroscience methods 2019, 311:402-407.

10. Barten DM, Cadelina GW, Hoque N, DeCarr LB, Guss VL, Yang L, Sankaranarayanan S, Wes PD, Flynn ME, Meredith JE, et al: Tau transgenic mice as models for cerebrospinal fluid tau biomarkers.Journal of Alzheimer's disease : JAD 2011, 24 Suppl 2:127-141.

11. Liu L, Duff K: A technique for serial collection of cerebrospinal fluid from the cisterna magna in mouse.Journal of visualized experiments : JoVE 2008.

12. Ineichen BV, Schnell L, Gullo M, Kaiser J, Schneider MP, Mosberger AC, Good N, Linnebank M, Schwab ME: Direct, long-term intrathecal application of therapeutics to the rodent CNS.Nature protocols 2017, 
12:104-131.

13. Jones LL, Tuszynski MH: Chronic intrathecal infusions after spinal cord injury cause scarring and compression.Microsc Res Tech 2001, 54:317-324.

14. Yaksh TL, Rudy TA: Chronic catheterization of the spinal subarachnoid space.Physiol Behav 1976, 17:1031-1036.

15. Mclntyre C, Saville J, Fuller M: Collection of cerebrospinal fluid from murine lateral ventricles for biomarker determination in mucopolysaccharidosis type IIIA.Journal of neuroscience methods 2019, 324:108314.

16. Nair KGS, Ramaiyan V, Sukumaran SK: Enhancement of drug permeability across blood brain barrier using nanoparticles in meningitis.Inflammopharmacology 2018, 26:675-684.

17. Cirrito JR, May PC, O'Dell MA, Taylor JW, Parsadanian M, Cramer JW, Audia JE, Nissen JS, Bales KR, Paul SM, et al: In vivo assessment of brain interstitial fluid with microdialysis reveals plaqueassociated changes in amyloid-beta metabolism and half-life. The Journal of neuroscience : the official journal of the Society for Neuroscience 2003, 23:8844-8853.

18. Lim NK, Moestrup V, Zhang X, Wang WA, Møller A, Huang FD: An Improved Method for Collection of Cerebrospinal Fluid from Anesthetized Mice.Journal of visualized experiments : JoVE 2018.

19. Simon MJ, Iliff JJ: Regulation of cerebrospinal fluid (CSF) flow in neurodegenerative, neurovascular and neuroinflammatory disease.Biochimica et biophysica acta 2016, 1862:442-451.

20. Oshio K, Watanabe H, Song Y, Verkman AS, Manley GT: Reduced cerebrospinal fluid production and intracranial pressure in mice lacking choroid plexus water channel Aquaporin-1.FASEB journal : official publication of the Federation of American Societies for Experimental Biology 2005, 19:76-78.

21. Barkovits K, Kruse N, Linden A, Tönges L, Pfeiffer K, Mollenhauer B, Marcus K: Blood Contamination in CSF and Its Impact on Quantitative Analysis of Alpha-Synuclein.Cells 2020, 9.

22. Irani DN: Cerebrospinal Fluid in Clinical Practice. Saunders; 2009.

23. Shinozaki M, Yasuda A, Nori S, Saito N, Toyama Y, Okano H, Nakamura M: Novel method for analyzing locomotor ability after spinal cord injury in rats: technical note.Neurologia medicochirurgica 2013, 53:907-913.

24. Kiyatkin EA, Wakabayashi KT: Parsing glucose entry into the brain: novel findings obtained with enzyme-based glucose biosensors.ACS chemical neuroscience 2015, 6:108-116.

25. Waritani T, Chang J, McKinney B, Terato K: An ELISA protocol to improve the accuracy and reliability of serological antibody assays.Methods X 2017, 4:153-165.

26. Bothwell SW, Janigro D, Patabendige A: Cerebrospinal fluid dynamics and intracranial pressure elevation in neurological diseases.Fluids and barriers of the CNS 2019, 16:9.

27. Zhang L, Hussain Z, Ren Z: Recent Advances in Rational Diagnosis and Treatment of Normal Pressure Hydrocephalus: A Critical Appraisal on Novel Diagnostic, Therapy Monitoring and Treatment Modalities.Current drug targets 2019, 20:1041-1057. 
28. Takeda S, Sato N, Rakugi H, Morishita R: Molecular mechanisms linking diabetes mellitus and Alzheimer disease: beta-amyloid peptide, insulin signaling, and neuronal function.Molecular bioSystems 2011, 7:1822-1827.

29. Takeda S, Sato N, Uchio-Yamada K, Sawada K, Kunieda T, Takeuchi D, Kurinami H, Shinohara M, Rakugi $\mathrm{H}$, Morishita R: Diabetes-accelerated memory dysfunction via cerebrovascular inflammation and Abeta deposition in an Alzheimer mouse model with diabetes.Proceedings of the National Academy of Sciences of the United States of America 2010, 107:7036-7041.

30. Garg T, Bhandari S, Rath G, Goyal AK: Current strategies for targeted delivery of bio-active drug molecules in the treatment of brain tumor.Journal of drug targeting 2015, 23:865-887.

31. Dworkin RH, O'Connor AB, Kent J, Mackey SC, Raja SN, Stacey BR, Levy RM, Backonja M, Baron R, Harke $\mathrm{H}$, et al: Interventional management of neuropathic pain: NeuPSIG recommendations. Pain 2013, 154:2249-2261.

32. Harris VK, Stark J, Vyshkina T, Blackshear L, Joo G, Stefanova V, Sara G, Sadiq SA: Phase I Trial of Intrathecal Mesenchymal Stem Cell-derived Neural Progenitors in Progressive Multiple Sclerosis.EBioMedicine 2018, 29:23-30.

33. Naylor E, Aillon DV, Gabbert S, Harmon H, Johnson DA, Wilson GS, Petillo PA: Simultaneous real-time measurement of EEG/EMG and L-glutamate in mice: A biosensor study of neuronal activity during sleep.Journal of electroanalytical chemistry (Lausanne, Switzerland) 2011, 656:106-113.

34. Fu K, Miyamoto Y, Otake K, Sumi K, Saika E, Matsumura S, Sato N, Ueno Y, Seo S, Uno K, et al: Involvement of the accumbal osteopontin-interacting transmembrane protein 168 in methamphetamine-induced place preference and hyperlocomotion in mice.Scientific reports 2017, 7:13084.

\section{Figures}



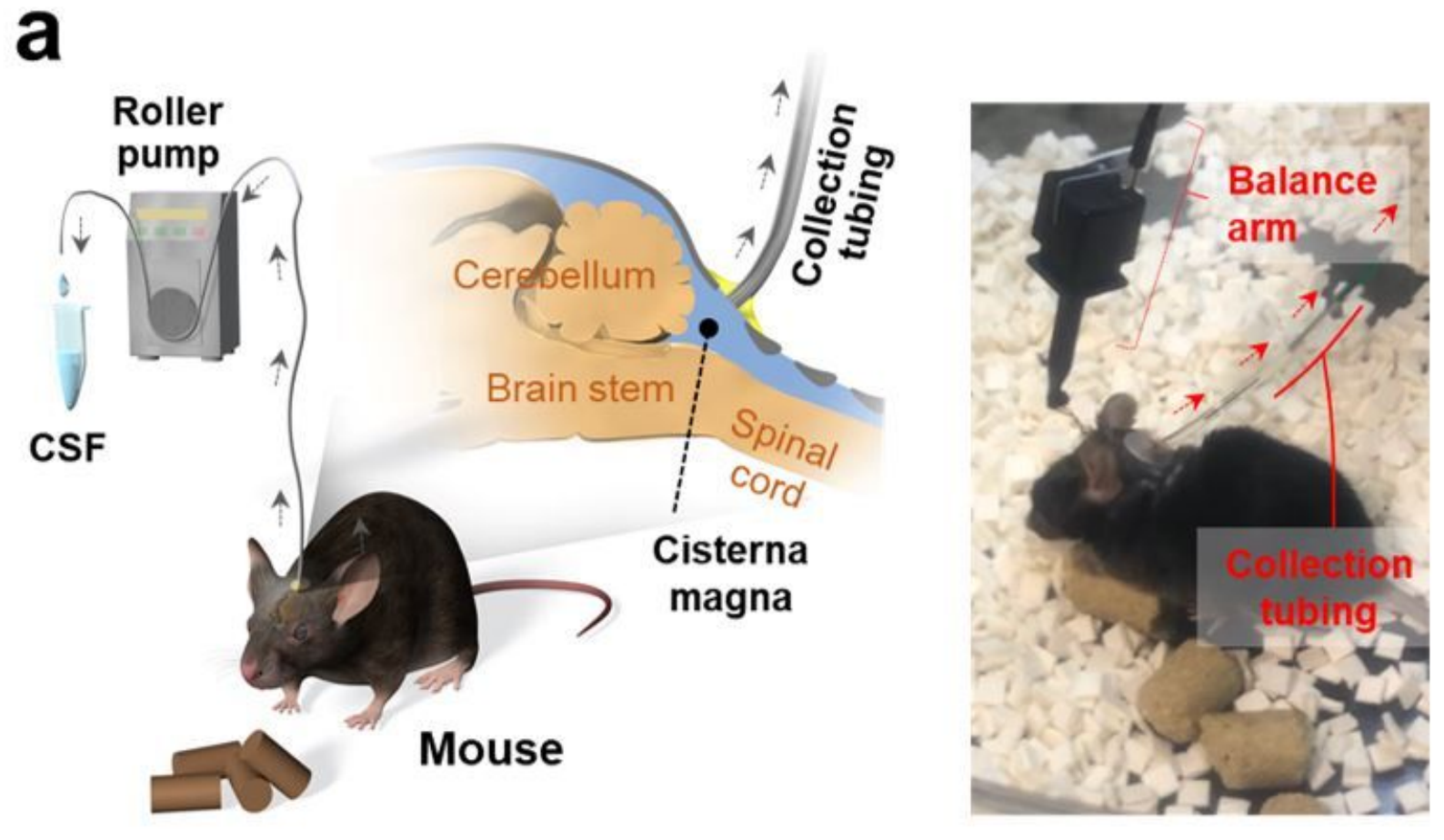

b
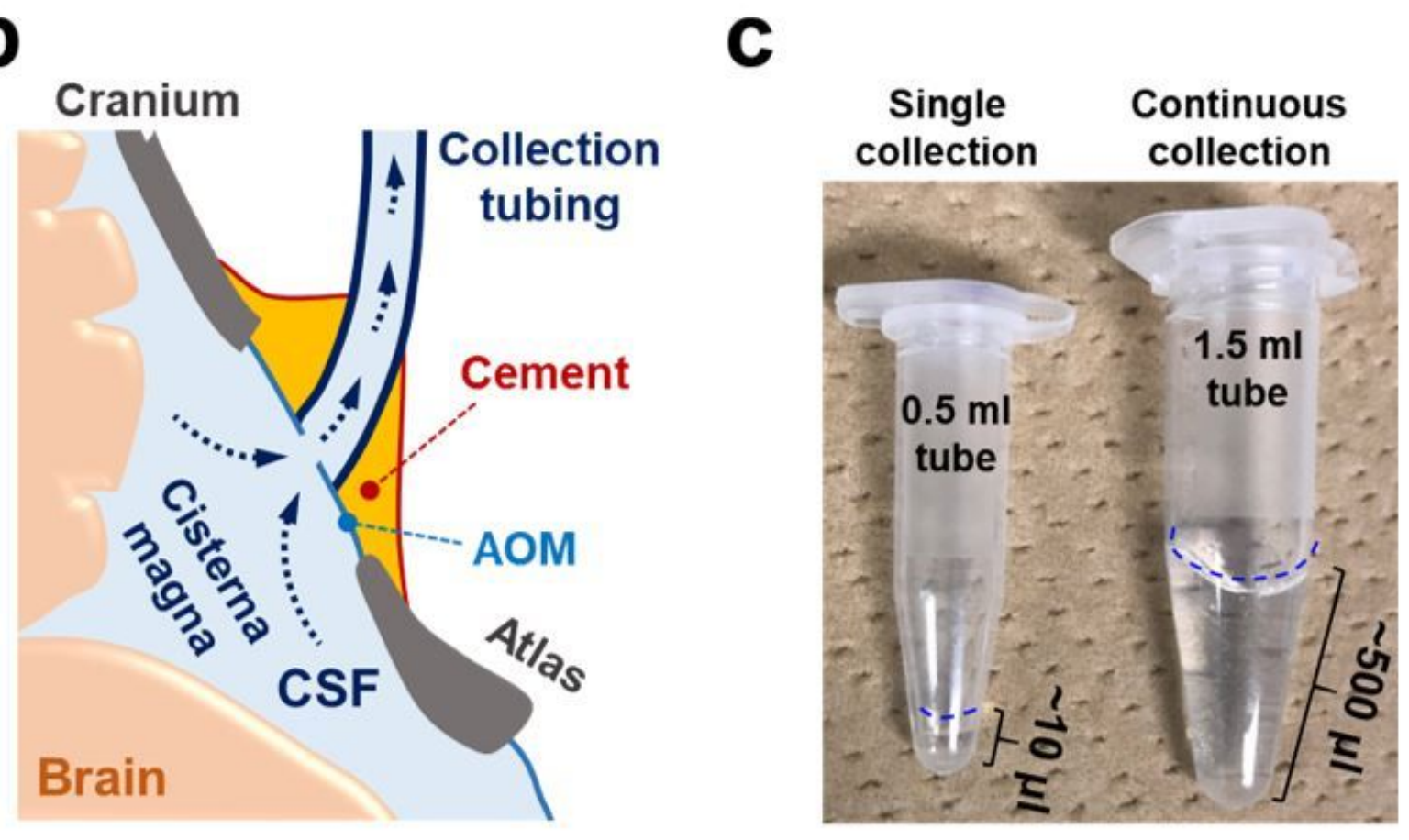

Figure 1

Continuous collection of large volume CSF in the free-moving mouse. (a) Schematic of continuous CSF collection in the free-moving mice (left panel). Mouse in the movement-response rotating cage (right panel). Mouse is connected to the sensor integrated balance arm to suspend and prevent twisting of the CSF tubing. (b) CSF tubing is fixed onto the AOM with dental cement covering caudal end of cranium, CSF tubing, and rostral end of the atlas. CSF is collected via small hole ( $0.2 \mathrm{~mm}$ of diameter, need to be 
smaller than the i.d. of the tubing) introduced on the AOM and through the CSF tubing connected to the pull continuous flow roller pump $(2-5 \mu \mathrm{l} / \mathrm{h})$. Note that the end of the CSF tubing does not enter the subarachnoid space. (c) A large volume of clear CSF collected from a single mouse during consecutive five days (right). Conventional single-collection method yields $\sim 10 \mu \mathrm{l}$ of CSF per mouse (left).
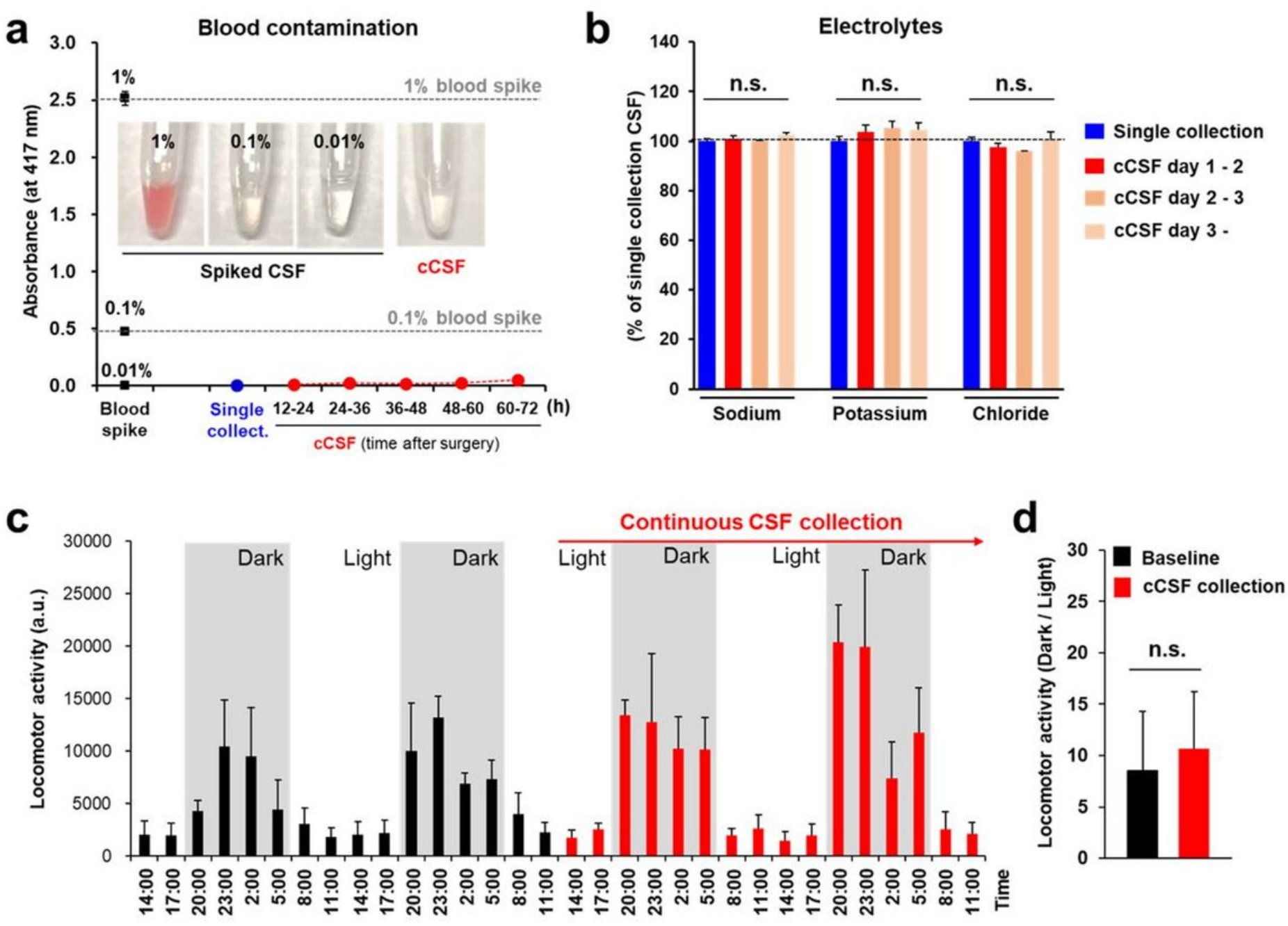

Figure 2

Continuous collection of high-quality CSF from naturally-behaving mice. (a) Quantitative assessment of blood contamination in CSF using highly sensitive spectrometer method. Levels of hemoglobin contamination (absorbance at $417 \mathrm{~nm}$ ) from blood spiked CSFs (intentionally spiked with $1,0.1$, or $0.01 \%$ $\mathrm{vol} / \mathrm{vol}$ of blood, black), mouse CSF collected by conventional single collection technique (blue), and CCSF at various time points after surgery (red). Insets are representative photo images of spiked CSF and cCSF. (b) Normal electrolyte concentrations in the cCSF during long-term continuous collection. Sodium, potassium, and chloride levels were measured in the single-collection CSF and CCSF at various time points after surgery ( $n=4-6$, one-way ANOVA). (c) Normal locomotor activities and circadian rhythms of the mice during long-term cCSF collection. Locomotor activities of the mice were measured every three hours before (baseline, black) and during continuous CSF collection (red) under the normal 12:12 h lightdark cycle. (d) The locomotor activity dark/light ratio during baseline period and continuous CSF 
collection is shown ( $n=3$, Student's t-test). CSF, cerebrospinal fluid; AOM, atlanto-occipital membrane; i.d., inner diameter, cCSF, CSF obtained by the continuous collection method; n.s., not significant.
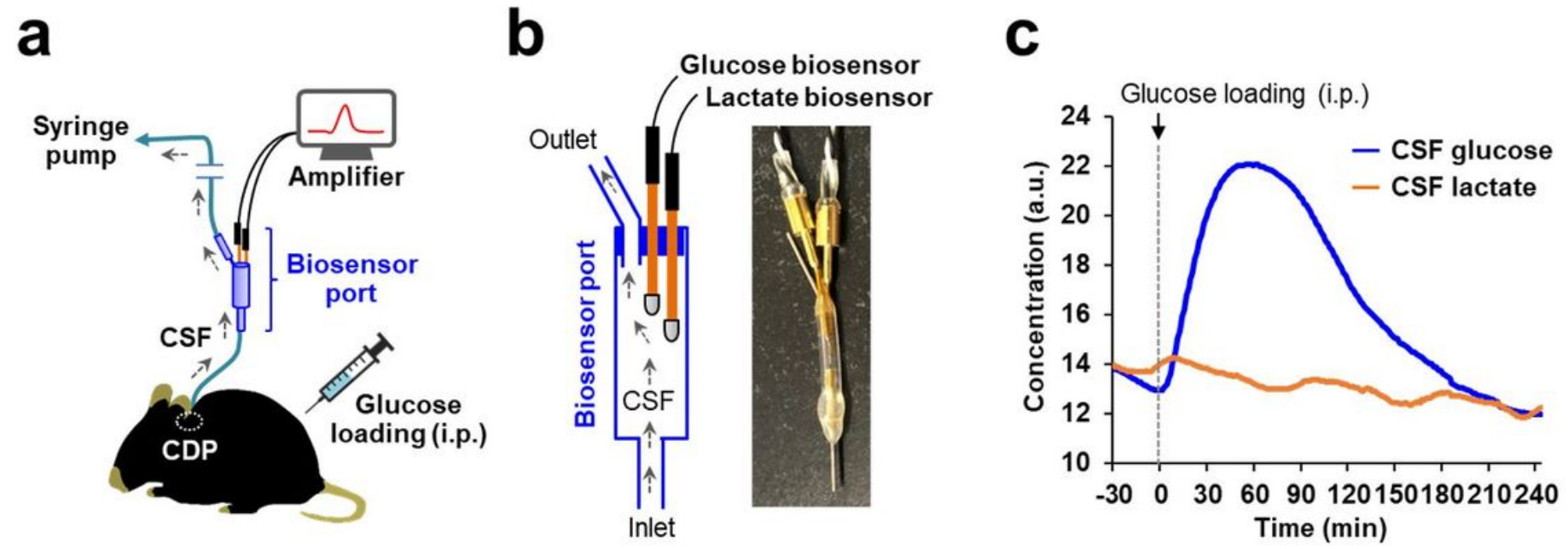

\section{Figure 3}

Minimal histological damage on brain and spinal cord after chronic dural port implantation. (a) Scheme of the sagittal view of the mouse brain and spinal cord area adjacent to CM of intact and sham-operated (top), CDP (middle), and chronic CMc (bottom). Dotted square indicates the areas where the histological appearances are shown in (b-c). (b, c) Histological integrity and astrocyte reactivity two weeks after CDP or chronic CMc implantation compared to the sham-operated mice. (b, top panels) H\&E-stained sagittal sections of the BS and spinal cord area adjacent to CM of sham-operated, CDP, and chronic CMc. Scale bar, $200 \mu \mathrm{m}$. (b, bottom panels) High magnification of the H\&E-stained sagittal sections corresponding to the dotted outlined area in the top panels. Scale bar, $50 \mu \mathrm{m}$. (c) GFAP-stained sagittal sections corresponding to the outlined area in the top panels of (b); high magnification of GFAP-positive cells in the areas outlined with white dotted square are shown as insets. Scale bar, $50 \mu \mathrm{m}$. (d) Quantification of GFAP-positive cells in the area corresponding to the dotted outlined area in the top panels of (b). $n=6$ / group. ${ }^{*} p<0.05$ versus naïve and CDP mice. One-way ANOVA and a subsequent Tukey-Kramer test. Data presented are the mean \pm SEM. BS, brain stem; $\mathrm{Cb}$, cerebellum, CDP, chronic dural port; CM, cisterna magna; CMc, cisterna magna cannulation; H\&E, haematoxylin-eosin; GFAP, glial fibrillary acidic protein. 
a

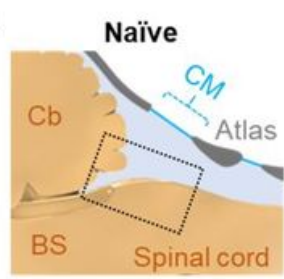

Chronic dural port

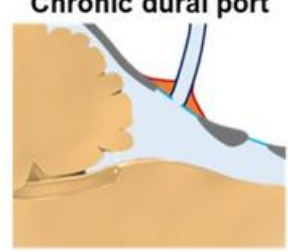

Chronic CMc

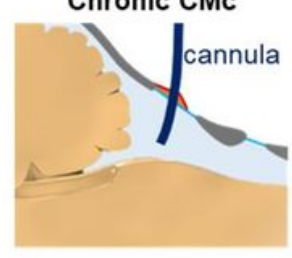

b

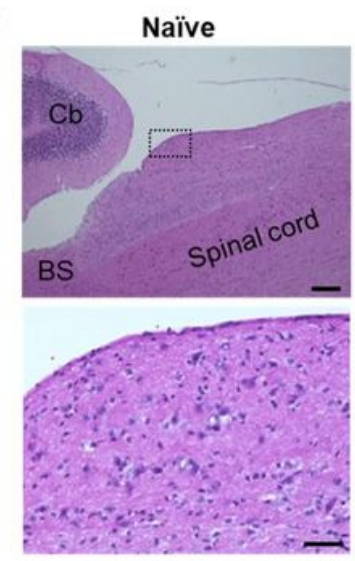

c

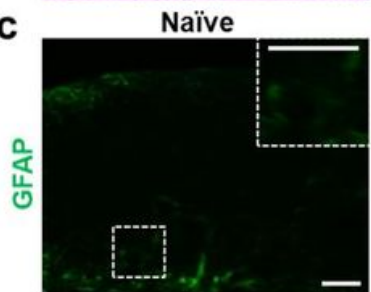

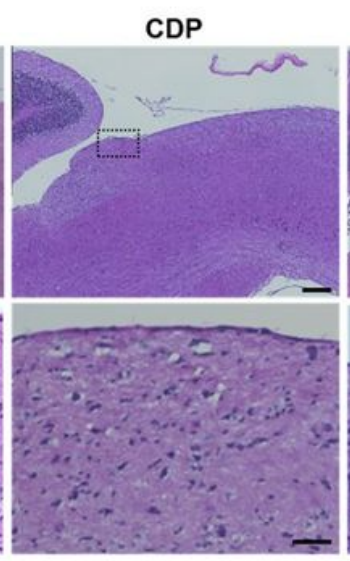

CDP

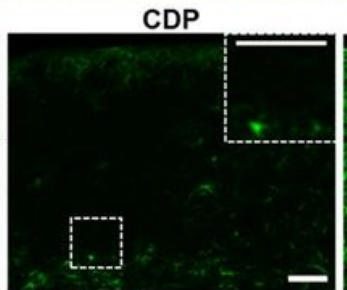

Chronic CMc

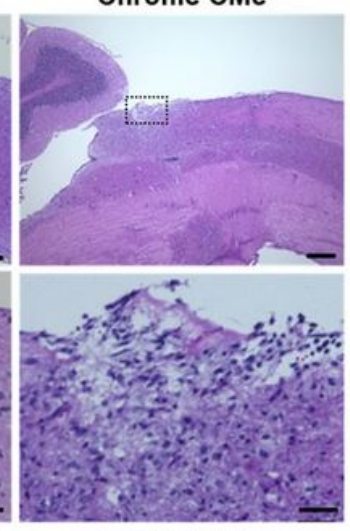

Chronic CMc

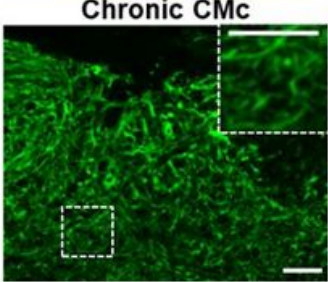

d

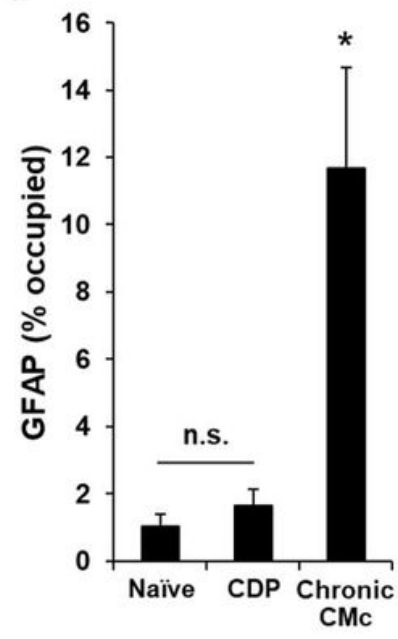

\section{Figure 4}

Monitoring real-time changes in CSF glucose concentration in the free-moving mice. (a) Schematic of continuous collection of CSF via CDP for monitoring glucose and lactate levels during glucose loading. Levels of glucose and lactate in CSF were continuously and quantitatively measured using biosensors attached in the middle of collection tubing (biosensor port). CSF was collected at the flow rate of 0.05 $\mu \mathrm{l} / \mathrm{min}$ using syringe pump. Mice were given an intraperitoneal injection of glucose ( $2 \mathrm{mg} / \mathrm{g}$ body weight) during continuous CSF collection. (b) Schematic of biosensor port. Two electrodes of glucose and lactate biosensors are installed within the port. Electrodes are exposed to the continuous flow of CSF and provide real-time changes in glucose and lactate concentrations. (c) CSF levels of glucose (blue line) and lactate (orange line) after intraperitoneal glucose loading. A representative data is shown from a wild-type mouse. CDP, chronic dural port; CSF, cerebrospinal fluid; i.p., intraperitoneal injection. 


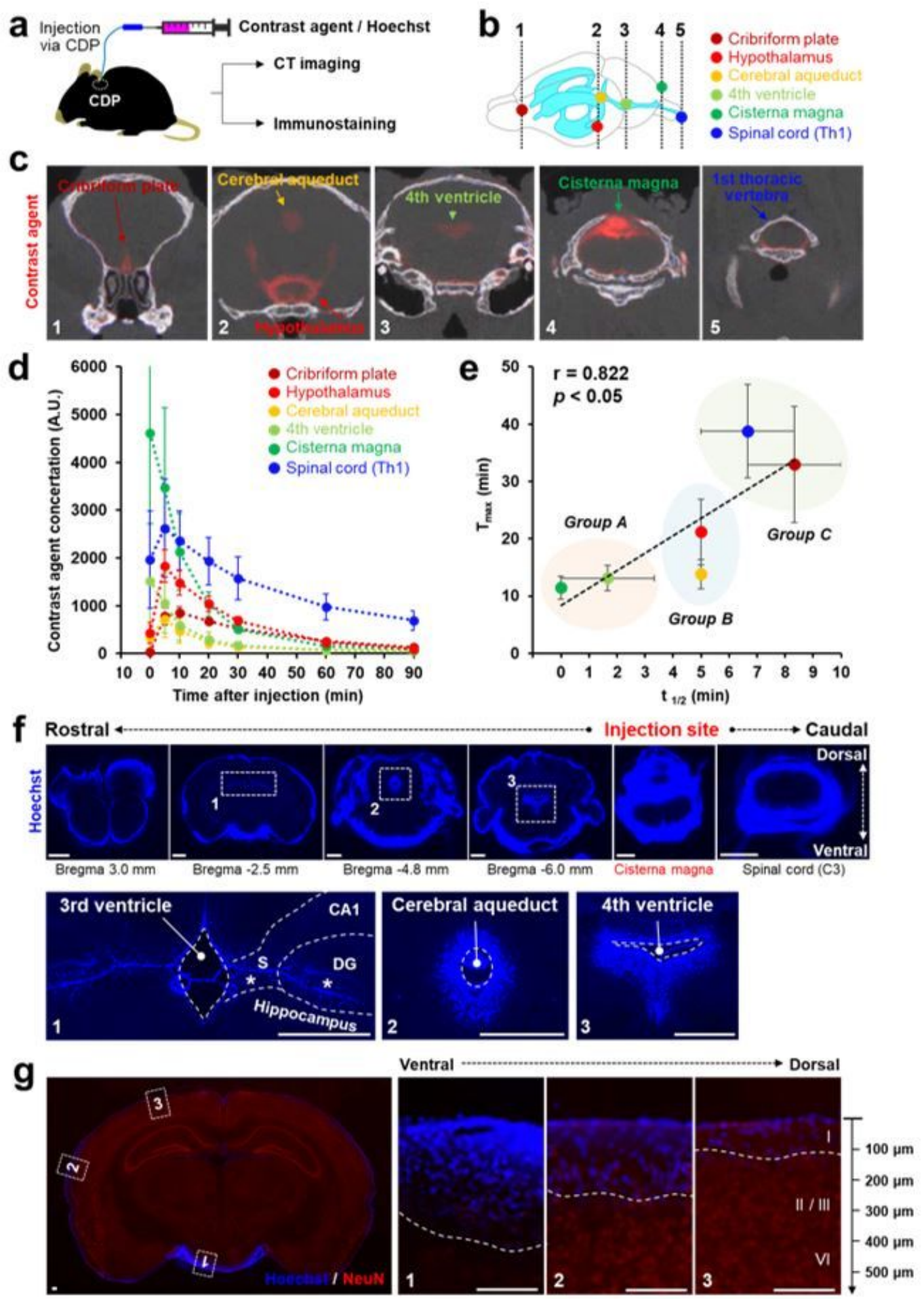

\section{Figure 5}

Injection of CSF tracer via CDP and its temporal and spatial distributions in mice. (a) Schematic of acute injection of tracers into CSF system in free-moving mice via CDP and subsequent analysis of tracers' dynamic distribution and metabolism by micro-CT imaging and brain sections. (b) Schematic of ventricles of mouse brain. (c) Representative coronal brain images of Micro-CT with contrast agent (shown in red). Numbers in the right bottom corner of each image are corresponding to the levels of 
sections indicated in (b). (d) Quantification of the concentrations of contrast agent in each region. Radiodensity values of contrast agent were sequentially measured at each time point. $n=3 /$ group. Data presented are the mean \pm SEM. (e) Relationship between half-life (t1/2) and Tmax values of contrast agents in each region. Each plot represents the mean of three animals with SEM error bars. $p<0.05$, Spearman rank test. Subregions were divided into three groups depending on the metabolic rates of tracers. Group A; regions with rapid distribution and fast clearance of the tracer; Group B; regions with intermediate metabolism of the tracer, Group C; regions with delayed distribution with slow clearance of the tracer. (f) Representative images of coronal sections of mouse brain after acute injection of Hoechst solution into cisterna magna (injection site) via CDP. (f, bottom panels) High magnification of the sections corresponding to the dotted outlined area in the top panels. Scale bar, 1,000 $\mu \mathrm{m}$. (g) Representative image of coronal section immunostained with neuronal marker NeuN. High magnification of the areas corresponding to the dotted outlined area in the right panel are shown in the left three panels. Cortical layers are indicated by Roman numerals in panel 3. Scale bar, $200 \mu \mathrm{m}$. CDP, chronic dural port; CT, computed tomography; DG, dentate gyrus; S, subiculum; Tmax, time to peak concentration. 


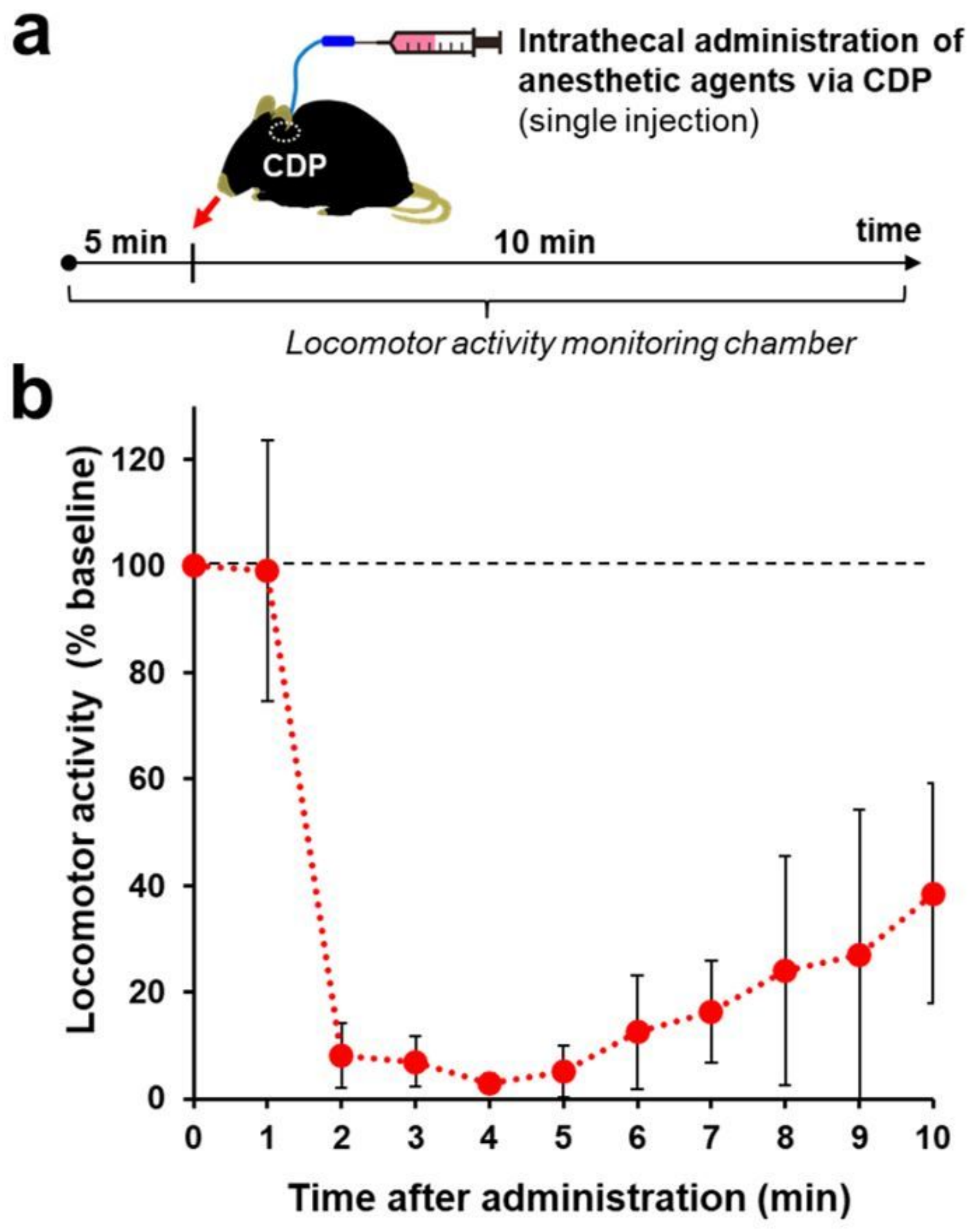

Figure 6

Acute intrathecal administration of drug via CDP and behavioral assessment in the free-moving mouse. (a) Time course of the experiment. Anesthetic agent (midazolam) was acutely injected via CDP in the free-moving mice ( $5 \mu \mathrm{l}$ in $1 \mathrm{sec}$ by syringe connected to CDP), while locomotor activities were quantitatively measured by infrared light beam crossing system. (b) Locomotor activity after intrathecal 
administration of anesthetic agent via CDP in the free-moving mice. $n=4$ / group. Data presented are the mean \pm SEM. CDP, chronic dural port. 